This is an author produced version of a paper published in European journal of oral sciences. This paper has been peer-reviewed but does not include the final publisher proof-corrections or journal pagination.

Citation for the published paper:

Brogårdh-Roth, Susanne; Klingberg, Gunilla; Matsson, Lars. (2014).

Mixed-up results give misguided conclusions regarding enamel defects in permanent teeth in children born preterm. European journal of oral sciences, vol. 122 , issue 5 , p. null

URL: https://doi.org/10.1111/eos.12146

Publisher: Wiley

This document has been downloaded from MUEP (https://muep.mah.se) / DIVA (https://mau.diva-portal.org). 


\section{Mixed-up results give misguided conclusions regarding enamel defects in permanent teeth in children born preterm}

\section{Dear Editor,}

We read with great interest a recent review article by JACOBSEN et al. (1), in which the authors described developmental enamel defects in children born preterm. The article is a welcome addition to the knowledge about dental issues in children born preterm. In this systematic review, the authors included follow-up studies on the association between prematurity, low birth weight, and developmental defects in the primary and permanent dentitions.

We were happy to see that one of our publications was included in the review, an article concerning developmental defects, including molar incisor hypomineralization (MIH) in the permanent dentition, in Swedish children born preterm (2). Unfortunately, when looking deeper into the article by JACOBSEN and co-workers (1) it is clear that our publication has not been cited correctly, and this has caused the results and conclusions drawn by JACOBSEN et al. to be affected in a most unfortunate way.

We refer to Table 3 in the review (1), where the authors provide a summary of the material, number of subjects and age, definition of exposure, methods, diagnostic criteria, teeth examined, findings and $p$-values from the included articles. For our publication (2), the frequencies quoted regarding DDE (developmental defects of the enamel), including MIH, have been mixed up with data for opacities. Data on all types of enamel defects were presented in the same Table 2 in our publication and, unfortunately, JACOBSEN et al. have misquoted those results.

In their Table 3, JACOBSEN et al. give the frequencies for DDE, including MIH, to $55 \%$ for 'exposed' and $44 \%$ for 'controls'. However, the frequencies were in fact $69.5 \%$ for 
'exposed' (preterm) and 51\% for 'controls' (full-term), resulting in a statistically significant difference between children born preterm and matched controls regarding DDE (2). Whereas the $p$-value should be 0.024 , as written in the original article, it is given as $p=0.2$ in the review by JACOBSEN et al. (1).

This mistake is very unfortunate, since the authors state in their conclusions that currently there is no evidence that being born prematurely influences development of the permanent teeth. This conclusion is thus based on their Table 3 with incorrect information on DDE, including MIH and permanent teeth (2). The authors must therefore reconsider the formulation of their conclusions.

In addition, the age of our subjects is listed by Jacobsen et al. in Table 3 as 9 to $12 \mathrm{yr}$ of age. However, this is also incorrect. The age should be 10 to $12 \mathrm{yr}$ as stated in the original article (2).

\section{References}

1. Jacobsen PE, Haubek D, Henriksen TB, ØstergaArd JR, Poulsen S. Developmental enamel defects in children born preterm: a systematic review. Eur J Oral Sci 2014; 122: 7-14.

2. BROGÅRDH-Roth S, MATSSON L, KLINGBERG G. Molar-incisor hypomineralization and oral hygiene in 10- to-12-yr-old Swedish children born preterm. Eur J Oral Sci 2011; 119: $33-39$.

Susanne Brogårdh-Roth Gunilla Klingberg Lars Matsson Department of Paediatric Dentistry Faculty of Odontology, Malmö University, Sweden E-mail: susanne.brogardh@mah.se 